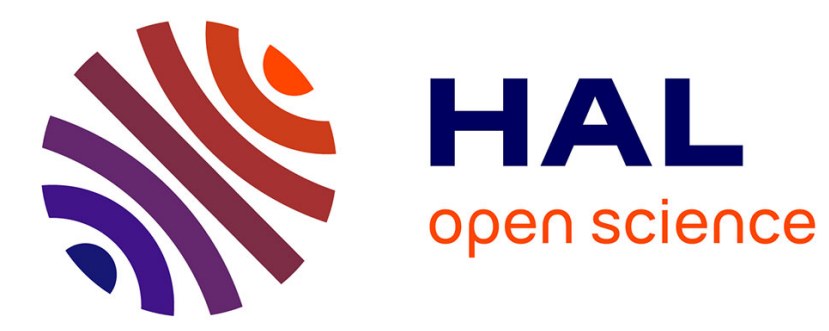

\title{
Les vestiges animaux du fossé gaulois de Beauvais : note préliminaire
}

\author{
Patrice Méniel
}

\section{To cite this version:}

Patrice Méniel. Les vestiges animaux du fossé gaulois de Beauvais: note préliminaire. Revue archéologique de Picardie, 1983, Les Celtes dans le nord du bassin parisien. Actes du Ve colloque de l'Association française pour l'étude de l'âge du Fer, 1, pp.226-228. 10.3406/pica.1983.3001 . hal02536390

\section{HAL Id: hal-02536390 \\ https://hal.science/hal-02536390}

Submitted on 8 Apr 2020

HAL is a multi-disciplinary open access archive for the deposit and dissemination of scientific research documents, whether they are published or not. The documents may come from teaching and research institutions in France or abroad, or from public or private research centers.
L'archive ouverte pluridisciplinaire HAL, est destinée au dépôt et à la diffusion de documents scientifiques de niveau recherche, publiés ou non, émanant des établissements d'enseignement et de recherche français ou étrangers, des laboratoires publics ou privés.

\section{(이) $\$$}

Distributed under a Creative Commons Attribution - NonCommercial - NoDerivatives| 4.0 


\title{
LES VESTIGES ANIMAUX DU FOSSÉ GAULOIS DE BEAUVAIS
}

\author{
Note préliminaire
}

\author{
par Patrice MENIEL *
}

Le fossé gaulois de Beauvais, en cours de fouille actuellement (février 1982), a déjà livré un ensemble de 2600 vestiges osseux animaux dans un état de conservation exceptionnel. En effet, la tourbe, qui constitue le remplissage de cette structure, nous les a remarquablement restitués. Mais ce milieu est très homogène, ce qui entraîne, lors de la fouille, la formation de mottes assez difficiles à briser. Un nombre important d'ossements a pu ainsi être oublié, et seul un tamisage peut nous renseigner sur l'importance de ce phénomène. La pratique du tamisage nous a permis, par ailleurs, de collecter une microfaune riche et variée: micromammifères, oiseaux, poissons, batraciens, coquillages et insectes, dont la présence n'est certainement pas due à l'homme. Cependant, les poissons ont pu être consommés avant d'être rejetés à l'état de déchets dans leur milieu naturel.

Les animaux sauvages sont, dans la plupart des cas, associés à un milieu particulier. Leur découverte permet donc de reconstituer partiellement l'environnement et de donner des indications quant à la couverture végétale. La liste des animaux sauvages que nous avons déterminée et les milieux où on les trouve habituellement sont regroupés dans le tableau I. Ces données permettent déjà de se faire une idée du milieu végétal aux alentours du fossé, mais cette image partielle (fig. 1) devra être complétée par les résultats des études botaniques.

Les animaux domestiques sont numériquement dominants (tab. II) : ils représentent $96 \%$ des vestiges déterminés. C'est le porc qui a fourni les plus grands nombres de restes et d'individus, puis le bœuf. Le mouton et la chèvre sont beaucoup moins nombreux, comme le montrent d'ailleurs les proportions calculées à partir des poids de viande fournis à l'alimentation (tab. III). Ce dernier tableau montre que l'alimentation carnée était bașée sur le porc et le bœuf, mais que d'autres animaux, comme le cheval et le chien, n'étaient pas dédaignés. Cependant, si la production de viande est attestée pour ces derniers animaux, elle n'est pas, dans la majorité des cas, la seule utilisation possible de ceux-ci. En effet, si on se place, par exemple, du point de vue de la garde assurée par les animaux, alors ce sont les chiens, mais aussi les oies, qui sont importants. Le classement des animaux change suivant les productions que l'on considère, mais ces dernières ne sont pas faciles à mettre en évidence.

En dehors de ces premiers aspects, les ossements permettront une description assez précise de la morphologie des animaux domestiques de ce site. En effet, plusieurs crânes et fragments de squelettes, intéressant l'étude zoologique, ont déjà été dégagés. De plus leur état de conservation exceptionnel sera un atout supplémentaire pour cette étude.

D'autre part, la collecte de nombreuses mâchoires de porcs, environ trois cents à I'heure actuelle, permettra d'étudier les âges d'abattages. Les premières déterminations montrent qu'une majorité d'animaux jeunes des deux sexes étaient abattus vers deux ans, alors que pour les adultes, il s'avère que les truies étaient conservées plus longtemps que les verrats.

Les âges d'abattage des ruminants sont en moyenne peu élevés; en effet, les animaux âgés sont rares. Ceci peut être le reflet d'un élevage orienté vers la production de viande, à moins que le milieu environnant ait réduit la longévité de ces animaux, comme cela est mentionné dans la Description du canton de Beauvais en 1851 par GRAVES (1).

Cette étude permettra donc de déterminer l'influence d'un milieu humide sur la faune d'un site gaulois. Cette influence se traduit par la présence d'une microfaune variée, et peut-être par des effets sur la morphologie et/ou sur la longévité des animaux domestiques qui représentent en fait la plus grande part de la faune. II sera inléressant de comparer ces effets à ceux observés sur des sites contemporains mais différents du point de vue du milieu environnant.

(1) GRAVES, L., Précis statistique du canton de Beauvais. Extrait de l'annuaire de 1851, réédition 1980. 
Tableau I. Espèces sauvages rencontrées et leurs milieux de prédilection

ESPÉCE

HABITAT
le cerf
la martre
le lièvre
le campagnol amphibie
le campagnol agreste
le rat des moissons
la musaraigne bicolore

forêts plus ou moins denses

dans les arbres

terrain découvert, terre cultivée, pâturages, marais

autour des rivières à cours lent, et des lacs aux berges bien

fournies en végétation

dans les prés et les pâturages humides

dans les marais couverts de roseaux

lisière des bois, dans une végétation dense

Tableau II. Liste des espèces animales présentes dans le fossé de Beauvais, "les Aulnes du Canada" — février 1982 -

\begin{tabular}{|c|c|c|c|c|c|}
\hline \multicolumn{2}{|c|}{ ESPĖCES ANIMALES } & \multirow{2}{*}{$\begin{array}{c}\text { NR } \\
1274\end{array}$} & \multirow{2}{*}{$\begin{array}{c}\% \\
49,0\end{array}$} & \multirow{2}{*}{$\begin{array}{l}\text { NMI } \\
224\end{array}$} & \multirow{2}{*}{$\begin{array}{c}\% \\
69,1\end{array}$} \\
\hline le porc & Sus domesticus & & & & \\
\hline le bœuf & Bos taurus & 697 & 26,8 & 40 & 12,3 \\
\hline le mouton et la chèvre & Ovis aries et Capra hircus & 274 & 10,5 & 26 & 8,0 \\
\hline \multicolumn{2}{|l|}{ les équidés } & 100 & \multirow[t]{3}{*}{3,8} & 10 & \multirow[t]{3}{*}{3,1} \\
\hline le cheval & Equus caballus & \multirow{2}{*}{$\begin{array}{r}(25) \\
(8)\end{array}$} & & \multirow{2}{*}{$\begin{array}{l}\text { (7) } \\
\text { (3) }\end{array}$} & \\
\hline l'âne & Equus asinus & & & & \\
\hline le chien & Canis familiaris & 86 & 3,3 & 5 & \multirow[t]{2}{*}{1,5} \\
\hline le cerf & Cervus elaphus & 11 & 0,4 & 3 & \\
\hline la martre & Martes martes & 59 & 2,3 & 1 & \multirow[t]{2}{*}{1,8} \\
\hline le lièvre & Lepus europaeus & 3 & 0,1 & 1 & \\
\hline le campagnol amphibie & Arvicola sapidus & 1 & \multirow{4}{*}{0,3} & 1 & \multirow{4}{*}{2,8} \\
\hline le campagnol agreste & Microtus agrestis & 3 & & 3 & \\
\hline le rat des moissons & Micromis minutus & 3 & & 3 & \\
\hline la musaraigne bicolore & Crocidura leucodon & 2 & & 2 & \\
\hline l'oie & Anser anser domesticus & 52 & 2,0 & 3 & \multirow{3}{*}{1,2} \\
\hline le coq & Gallus gallus & 3 & 0,1 & 1 & \\
\hline \multicolumn{2}{|l|}{ oiseaux indéterminés } & 4 & 0,1 & & \\
\hline \multirow{2}{*}{\multicolumn{2}{|c|}{$\begin{array}{l}\text { batraciens indéterminés } \\
\text { poissons indéterminés }\end{array}$}} & 16 & 0,6 & & \\
\hline & & 10 & 0,3 & & \\
\hline \multicolumn{2}{|l|}{ 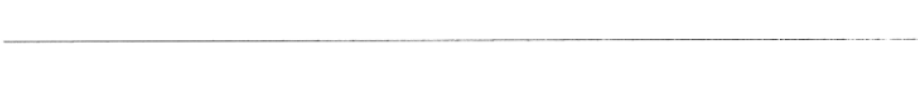 } & 2598 & & 323 & \\
\hline
\end{tabular}




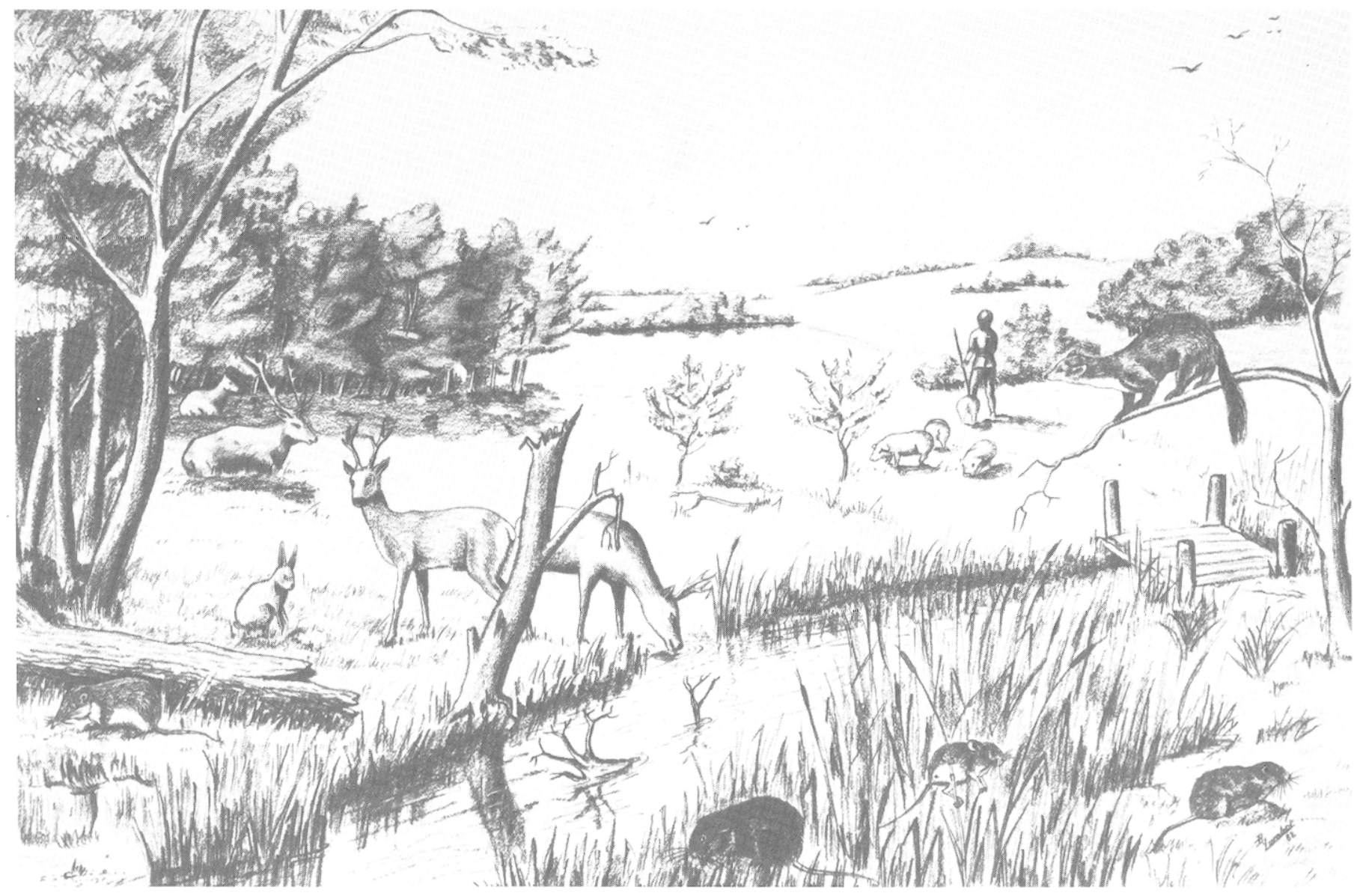

Fig. 1 : Essai de reconstitution de l'environnement à partir des premiers résultats de tamisage (dessin B. Lambot).

Tableau III. Rapport entre les différentes espèces domestiques, du point de vue de la production de viande

\begin{tabular}{lcrcc}
\hline & $\begin{array}{c}\text { poids de viande } \\
\text { par individus (kg) }\end{array}$ & NMI & $\begin{array}{c}\text { poids total } \\
\text { (kg) }\end{array}$ & $\%$ \\
\hline le porc & 50 & 224 & 11200 & 64 \\
le bœuf & 100 & 40 & 4000 & 23 \\
le mouton et la chèvre & 20 & 26 & 520 & 3 \\
le cheval & 200 & 7 & 1400 & 2 \\
l'âne & 90 & 3 & 270 & - \\
le chien & 7 & 5 & 35 & - \\
I'oie & 2 & 3 & 1 & - \\
le coq & 1 & 1 & 17432 & \\
\hline
\end{tabular}

